\title{
CONSTRUCTION MAXIMUM LIFETIME TREE AND ADAPTATION IN WIRELESS SENSOR NETWORKS
}

\author{
Yong Chan Lee ${ }^{1}$, Yong Hak Sin ${ }^{2}$ and Won Chol Jang ${ }^{3}$ \\ ${ }^{1}$ College of Information Science, Kim Il-sung University, Democratic People's Republic of Korea \\ ${ }^{2,3}$ Institute of Information Science, Kim Il-sung University, Democratic People's Republic of Korea
}

\begin{abstract}
The maximum lifetime problem in wireless sensor network is important to monitor a set of interesting target locations and route the collected information to a central base station. In this paper, first, we consider the method of construction maximum lifetime tree taking into account general type of data aggregation, exchange of control messages and packet transmission loss. Second, we consider the method increasing lifetime of tree and reducing complexity and latency combining optimization of energy consumption in entire network through quasioptimization of local nodes and adapting. Experiment results show that the proposed method is more robust and valid than the previous method.
\end{abstract}

Keywords:

Wireless Sensor Network (WSN), Maximum Lifetime, Data Gathering Tree

\section{INTRODUCTION}

In general, wireless sensor network (WSN) consists of a large number of sensor nodes and a sink deployed over sensing area. Every sensor node gathers data and sends data to sink by multihop communication and the sink is connected to master computer. Usually gathered data is sent from nodes to sink by clustering, since it is not efficient in terms of energy for every node to send the gathered data directly to sink $[1,3]$.

The research has been widely performed for constructing the maximum lifetime tree in WSN. The network lifetime maximization techniques have attracted a lot of research attention owing to their importance in terms of extending the flawless operation of battery-constrained WSNs [6]. Construction maximum lifetime tree can be divided into two categories: with aggregation and without aggregation $[2,4,5]$.

Problem formatting minimizing objective function, reciprocal of lifetime is common in construction maximum lifetime tree in previous works. Also, only energy consumed on transmitting and receiving data is considered and energy consumed on calculation and latency is not considered. Moreover energy consumed on exchange of control messages is not considered at all. Some experiment results show that energy consumed on exchange of control messages is at most 30 50\% of energy consumed on exchange of data message [2].

Although it is assumed that there is no loss of data packets during transmitting, experiment results show that about $30 \%$ of packets are lost in the middle of transmitting. Also, it has been considered that the network dies, even if a node of network is died. So, only reducing energy consumption of node with minimum lifetime has been considered and optimization of energy consumption in entire network has not been considered.

In this paper, first, we propose evaluation equations of lifetime of network considering exchange of control messages and transmitting loss and construction maximum lifetime tree based on it in data gathering WSN. Second, we propose construction maximum lifetime tree using adapting tree based on residual energy of sensor nodes. Third, we analyze and evaluate the validity of the proposed methods.

\section{PROPOSED METHOD}

\subsection{CONSTRUCTION MAXIMUM LIFETIME TREE IN WSN}

In this paper, we referred data gathering WSN, sensor nodes gather and transmit two kinds of data messages every period: one is a data message that can be aggregated and another is a data message that can't be aggregated such as control message.

\section{Assumption:}

1. WSN is static.

2. Every node has limited energy and computability and the sinks are powered.

3. Every node has identical transmitting and sensing are and lifetime of network is specified by lifetime of node with minimum lifetime.

4. The network is scheduled so that energy consumed on packet collision, latency and overhearing is 0 .

Following mathematical notations are used through this paper. The number of nodes in WSN is $N$ and nodes are noted by $v_{1}, v_{2}, \ldots, v_{N}$.

The sink is noted by $v_{0} . E_{i}$ is initial energy node $v_{i}, E_{i}^{(n)}$ is residual energy of node $v_{i}$ after $n$ periods, $E_{t}$ is energy consumption on which nodes transmit 1-bit, $E_{r}$ is energy consumption on which nodes receive 1-bit energy, $D_{i}(T)$ is degree of node $v_{i}$ in tree $T, C_{i}(T)$ is the number of children nodes of node $v_{i}$ in tree $T, \rho$ is a probability of transmission loss when transmitting $1 \mathrm{bit}, l_{1}$ is the length of aggregative data message that node gathers during one period (Type 1 ),$l_{2}$ is the length of not aggregative data message that node gathers during one period (Type 2), $G$ is the network of sensor nodes, $T$ is a tree belong to $G, T(S)$ is a set of trees creatable in $G, l_{c}$ is the length of control message, $E_{c}$ is energy consumed on calculating 1-bit, $E_{g}$ is energy consumed on gathering 1-bit, $E_{h}$ is energy consumed on receiving and overhearing 1-bit, $l_{2}(i, j)$ is the length of Type 2 data message in $j^{\text {th }}$ children node of $i^{\text {th }}$ node $v_{i}, l_{2}(i, 0)$ is the length of Type 2 data message that $i^{\text {th }}$ node $v_{i}$ gathered., $S(i, T)+1$ is the number of total nodes of subtree $T_{i}$ of which root is $v_{i}$ in tree $T$ and $E_{i}^{(0)}(k)$ is initial energy of node $v_{i}$ after adapting and changing a tree $k$ times. 


\subsubsection{Construction Maximum Lifetime Tree considering Exchange of Control Messages:}

We consider the case there are data that can be aggregated and not at the same time. Let us consider a tree $T$ of sensor nodes in WSN. Since $T$ is a tree, following Eq.is written in all nodes except for the sink.

$$
C_{i}(T)=D_{i}(T)-1
$$

During one period, node $v_{i}$ receives one of Type- $1 l_{1}$ bits messages from $C_{i}(T)$ children nodes. Then node $v_{i}$ aggregates above messages and its Type- 1 message, makes one of Type- $1 l_{1}$ bits messages and transmits to its parent node. Also, during one period, node $v_{i}$ receives one of Type- $2 l_{2}(i, j)$ bits messages from $C_{i}(T)$ children nodes.

Then node $v_{i}$ aggregates above messages and its Type-2 message, makes one of Type- $2 \sum_{j} l_{2}(i, j)$ bits messages and transmits to its parent node. So, energy consumed on transmitting and receiving data message during one period is as following.

$$
\begin{aligned}
& E_{\text {comdata }}(i)=C_{i}(T) l_{1} E_{r}+l_{1} E_{t}+\sum_{j=1}^{C_{i}(T)} l_{2}(i, j) E_{r}+\sum_{j=0}^{C_{i}(T)} l_{2}(i, j) E_{r} \\
& =E_{r}\left(l_{1} C_{i}(T)+\sum_{j=1}^{C_{i}(T)} l_{2}(i, j)\right)+E_{t}\left(l_{1}+\sum_{j=0}^{C_{i}(T)} l_{2}(i, j)\right)
\end{aligned}
$$

At this time, energy consumed on calculation is as following.

$$
E_{c a l}(i)=\left[\left(C_{i}(T)+1\right) l_{1}-1\right] E_{c}
$$

Also, energy consumed on data gathering itself is as following.

$$
E_{g a t}(i)=\left(l_{1}+l_{2}\right) E_{g}
$$

If there is no packet loss, parent nodes send one control message during one period, since they have no need to send respond message to messages of their children nodes. So, energy that node $v_{i}$ consumed on transmitting and receiving control message during one period is as follows:

$$
E_{\text {com }, \text { cont }}(i)=l_{c} E_{r}+C_{i}(T) l_{c} E_{t}
$$

Thus, energy that node $v_{i}$ consumed on transmitting and receiving control message, calculation and data gathering during one period is as follows:

$$
\begin{aligned}
E_{c o n}(i) & =E_{\text {com,data }}(i)+E_{\text {cal }}(i)+E_{\text {com }, \text { cont }}(i)+E_{\text {gat }}(i) \\
& =E_{r}\left[C_{i}(T) l_{1}+l_{c}+\sum_{j=1}^{C_{i}(T)} l_{2}(i, j)\right] \\
& +E_{t}\left[l_{1}+\sum_{j=0}^{C_{i}(T)} l_{2}(i, j)+C_{i}(T) l_{c}\right] \\
& +E_{c}\left[\left(C_{i}(T)+1\right) l_{1}-1\right] \\
& +\left(l_{1}+l_{2}\right) E_{g}
\end{aligned}
$$

So, lifetime of node $v_{i}$ in a given tree $T$ is as,

$$
\begin{aligned}
L(T, i)=\frac{E(i)}{E_{c o n}(i)}= & \frac{E(i)}{E_{r}\left[C_{i}(T) l_{1}+l_{c}+\sum_{j=1}^{C_{i}(T)} l_{2}(i, j)\right]+} \\
& E_{t}\left[l_{1}+\sum_{j=0}^{C_{i}(T)} l_{2}(i, j)+C_{i}(T) l_{c}\right]+ \\
& E_{c}\left[\left(C_{i}(T)+1\right) l_{1}-1\right]+\left(l_{1}+l_{2}\right) E_{g}
\end{aligned}
$$

The Eq.(6) is generalized Eq.in [2]. The problem of construction maximum lifetime tree is formatted as following.

$$
\arg \max _{T \in T(S)} \min _{i} L(T, i)
$$

It is much easier to optimize the reciprocal of $L(T, i)$ than $L(T, i)$ like in [2]. Because control parameters are on denominator in $L(T, i)$, but they are on numerator in $1 / L(T, i)$. So, Eq.(7) is changed by Eq.(9), defining that following Eq.as the weight of node $v_{i}$ in a tree $T$.

$$
\begin{aligned}
\omega(T, i)= & \frac{1}{L(T, i)} \\
= & \left\{\begin{array}{l}
E_{r}\left[C_{i}(T)\left(l_{1}+l_{2}\right)+l_{c}\right] \\
+E_{t}\left[l_{1}+l_{2}+C_{i}(T)\left(l_{1}+l_{c}\right)\right] \\
+E_{c}\left[\left(C_{i}(T)+1\right) l_{1}\right.
\end{array}\right\} \\
& \arg \min _{T \in T(S)} \max _{i} \omega(T, i)
\end{aligned}
$$

As seen in Eq.(8), in $\omega(T, i)$ is decided by $C_{i}(T)$ in a given WSN. From above equations, it can see easily that optimal tree is a tree that $C_{i}(T)$ is approximately proportional to $E(i)$. According to previous researches, this construction tree is NP-complete.

\subsubsection{Construction Maximum Lifetime Tree considering Packet Loss:}

Packet loss is not considered in construction maximum lifetime tree in most of previous works, but in practice it reaches average $30 \%$ [2]. From this, Construction maximum lifetime tree considering packet loss is referred in this paper.

If the probability of transmission failure transmitting 1-bit is $\rho$, the probability of transmission success transmitting 1 bits message is as following.

$$
(1-\rho)^{l} \approx 1-\rho \cdot l(\rho<<1)
$$

Since $\rho<<1$, Eq.10 is of approximate accuracy.

As seen in above equation, it can be seen that average $1 /(1-\rho l)$ messages should be sent in order to send one message exactly. During one period, node $v_{i}$ should receive one of Type- $1 l_{1}$ bits message from $C_{i}(T)$ children nodes.

However, average $\rho l_{1} C_{i}(T)$ messages are not received exactly, $\rho l_{1} C_{i}(T)$ control messages requiring retransmission should be sent to $\rho l_{1} C_{i}(T)$ nodes. The probability that these control messages are lost during transmission is $\rho l_{C}$. Since latency is one of important indexes, node $v_{i}$ requires no retransmission more.

Then node $v_{i}$ aggregates $\rho l_{1} \cdot \rho l_{C} \cdot \rho l_{1} \cdot C i(T)$ messages received next, $\left(1-\rho l_{1}\right) C i(T)$ messages received already, its message and transmits Type- $1 l_{1}$ bits message. This message also is required to retransmit with probability $\rho l_{1}$ in parent node and requiring message retransmission should be retransmitted with probability 
$\rho l_{1}$. It is assumed that there is only a require retransmission through this paper.

Energy consumed on transmitting and receiving Type-1 data messages, calculation, latency and gathering is as following.

$$
\begin{aligned}
& E_{\text {consum }, 1}(i, T)=\left(1-\rho l_{1}\right) l_{1} C_{i}(T) E_{r}+\rho l_{1} l_{1} C_{i}(T) E_{h} \\
& +\rho l_{1} C_{i}(T) l_{c} E_{t}+\left(\rho l_{1}\right)^{2} \rho l_{c} C_{i}(T) l_{1} E_{r} \\
& +\left[\left(1-\rho l_{1}\right)+\left(\rho l_{1}\right)^{2} \rho l_{c} l_{1} C_{i}(T)+l_{1}-1\right] E_{c}+l_{1} E_{g}+l_{1} E_{t}
\end{aligned}
$$

In case of Type-2 data message, energy consumed on transmitting and receiving, latency and gathering is same as case of Type-1 formally, but there is no energy consumed on data aggregating.

Let us $S(i, T)$ as the number of total nodes except for node $v_{i}$ in subtree $T_{i}$ of which root is $v_{i}$ in a tree $T$. Then Eq.(5)-Eq.(8) is as following.

$$
\sum_{j=1}^{C_{i}(T)} l_{2}(i, j)=l_{2} S(i, T), \sum_{j=0}^{C_{i}(T)} l_{2}(i, j)=l_{2}[S(i, T)+1]
$$

During one period, node $v_{i}$ should receive one of Type-2 $l_{2}(i, j)$ bits messages from $C_{i}(T)$ children nodes $\left(j=1, \overline{C_{i}(T)}\right)$. Since every message is lost with probability $\rho l_{2}(i, j)$, node $v_{i}$ sends following number of control messages requiring retransmission.

$$
\sum_{j=1}^{C_{i}(T)} \rho l_{2}(i, j)=\rho l_{2} S(i, T)
$$

Every node receives these messages with probability $\rho l_{c} \cdot \rho l_{2}(i, j)$, and retransmits Type $2 l_{2}(i, j)$, bits messages. These messages arrive at node $v_{i}$ of $l_{2}(i, j)$.

The node $v_{i}$ aggregates messages received initially, messages received again and its messages.

Then it sends up $l_{2} \sum_{j=1}^{C_{i}(T)}\left(1-\rho l_{2}(i, j)\right)+l_{2} \sum_{j=1}^{C_{i}(T)} \rho^{3} l_{c} l_{2}^{2}(i, j)+l_{2}$ bits messages to its parent node. That is, energy consumed on transmitting and receiving, calculation, latency and gathering is as following.

$$
\begin{aligned}
E_{\text {consum }, 2}(i, T) & =\sum_{j=1}^{C_{i}(T)}\left(1-\rho l_{2}(i, j)\right) l_{2} E_{r}+\sum \rho l_{2}(i, j) l_{2} E_{h} \\
& +\rho l_{2} S(i, T) l_{2} E_{t}+\sum_{j=1}^{C_{i}(T)} \rho^{3} l_{c} l_{2}^{2}(i, j) E_{r}+l_{2} E_{g} \\
& +l_{2}\left[1+\sum_{j=1}^{C_{i}(T)}\left(1-\rho l_{2}(i, j)\right)+\sum_{j=1}^{C_{i}(T)} \rho^{3} l_{c} l_{2}^{2}(i, j)\right] E_{t}
\end{aligned}
$$

Using Eq.(2), above Eq.(14) can be written as follows:

$$
E_{\text {consum }, 2}(i, T)=\left[\begin{array}{l}
l_{2} E_{r}\left(C_{i}(T)-\rho l_{2} S(i, T)\right) \\
+\rho l_{2}^{2} S(i, T) E_{h}+\rho l_{2}^{2} S(i, T) E_{t} \\
+\rho^{3} l_{c}\left(E_{r}+E_{t}\right) \sum_{j=1}^{C_{i}(T)} l_{2}^{2}(i, j) \\
+l_{2} E_{g}+l_{2} E_{t}+l_{2}\left[C_{i}(T)-\rho l_{2} S(i, T)\right] E_{t}
\end{array}\right]
$$

$$
\begin{aligned}
& =\left[\begin{array}{l}
{\left[C_{i}(T)-\rho l_{2} S(i, T)\right]\left[E_{t}+E_{r}\right] l_{2}} \\
+\rho l_{2}^{2} S(i, T)\left[E_{t}+E_{h}\right]+l_{2}\left[E_{g}+E_{t}\right] \\
+\left[E_{r}+E_{t}\right] \rho^{3} l_{c} \sum_{j=1}^{C_{i}(T)} l_{2}^{2}(i, j)
\end{array}\right] \\
& =\left[\begin{array}{l}
\left(E_{r}+E_{t}\right)\left[l_{2}\left(C_{2}(T)-\rho l_{2} S(i, T)\right)\right. \\
\left.\left.+\rho^{3} l_{c} \sum_{j=1}^{C_{i}(T)} l_{2}^{2}(i, j)\right)\right] \\
+\left(E_{t}+E_{h}\right) \rho l_{2}^{2} S(i, T)+\left(E_{g}+E_{t}\right) l_{2}
\end{array}\right]
\end{aligned}
$$

Energy that the node $v_{i}$ consumes during one period is as following.

$$
E_{\text {con }}^{\text {los }}(i)=E_{\text {consum }, 1}(i, T)+E_{\text {consum }, 2}(i, T)
$$

The lifetime of node $v_{i}$ in a given tree $T$ is

$$
L^{\text {los }}(T, i)=\frac{E(i)}{E_{c o n}^{\text {los }}(i)}
$$

The problem of construction maximum lifetime tree considering packet loss is formatted as following.

$$
\arg \max _{T \in T(S)} \min _{i} L^{\text {los }}(T, i)
$$

Since control parameter $C_{i}(T)$ is on denominator, defining $\omega^{\text {los }}(T, i)=\left(L^{\operatorname{los}}(T, i)\right)^{-1}$ as the weight of node $v_{i}$ in a tree $T$ considering packet loss, Eq.(18) is changed as following.

$$
\arg \min _{T \in T(S)} \max _{i} \omega^{\text {los }}(T, i)
$$

As seen in above equation, it can be seen that $\omega^{\text {los }}(T, i)$ is decided by $C_{i}(T)$ and construction maximum lifetime tree is a process searching a tree that $C_{i}(T)$ is proportional to $E(i)$ in a given WSN. This problem is NP-complete.

\subsection{CONSTRUCTION MAXIMUM LIFETIME TREE THROUGH APPROXIMATION ALGORITHM AND ADAPTING TREE}

\subsubsection{Approximation Algorithm:}

Since above formatting equations are NP-complete, complexity of these problem should be reduced using approximation algorithm. Formatting of the proposed problem in this paper is a generalization of [2], approximation algorithm can be referred as a combination of generalized MDST (Minimum Degree Spanning Tree) and MITT (Maximum lifetime Tree construction for data gathering without aggregation).

First, choose a tree $T$ randomly in WSN. Random tree $T$ is created as following:

Step 1: the first level node selects its neighbor nodes as its children nodes, about all children nodes of the sink $v_{0}$. At this time, it selects so that children nodes being shared one of nodes are same.

That is, the children node being shared selects the first level node as its parent node. All children nodes of the first level nodes selected in this way are called the second level nodes.

Step 2: the second level node selects all neighbors that are not the first and second level as its children nodes. The children node 
of the second level node is called the third level node.

Step 3: repeat step 2 about all neighbor nodes that are not $j^{\text {th }}$ level nodes.

Step 4: if all nodes are contained, output a tree $\mathrm{T}$ as random tree.

Calculating weights in every node in a selected tree $\mathrm{T}$, the nodes are classified into following three categories according to the weight.

Step 1: $V_{1}=\left\{v_{i} \mid \omega(T)-\phi<\omega(T, i) \leq \omega(T), v_{i} \in V\right\}$

where $V$ is the set of entire nodes. This node is called "the neck of a bottle" node.

Step 2: $V_{2}=\left\{v_{i} \mid \frac{\omega(T)-\phi-1}{E\left(v_{i}\right)}<\omega\right\}$

If the number of children nodes is increased by one, these nodes are called "the neck of a bottle" node. These nodes are quasi-"the neck of a bottle" node.

Step 3: $V_{3}=V-V_{1}-V_{2}: V_{3}$

The weight of a tree is as following.

$$
\omega(T)=\max _{i} \omega(T, i) \ldots(i=1, \bar{N})
$$

where, $\phi$ is the smallest range of change as follows.

$$
\phi=\frac{1}{E_{\max }}, E_{\max }=\max _{i} E(i)(i=1, \bar{N})
$$

These are not "the neck of a bottle" nodes, if the number of children nodes is increased by one.

Algorithm until now is same as in MDST and MITT. Other thing is only that weight calculation equations are different from them. In order to construct maximum lifetime tree, following equation is satisfied about all $i$ ideally.

$$
\omega(T, i)=\omega(T)
$$

However, since it cannot be done so, children nodes of "the neck of a bottle" nodes should be sent to children nodes of other "rich node" reducing children nodes of "the neck of a bottle" nodes as possible.

Step 1: find out $v_{j}$ that is adjacent to children node $v_{i}$ of "the neck of a bottle" node $v_{b}$, but is not children node of $v_{b}$.

If $v_{j}$ is a rich node, $v_{i}$ can be a children node of $v_{j}$. If $v_{j}$ is a children node of $v_{b}$, it can be local optimization, but cannot be optimization in entire network (Fig.1) because of following reasons.

As seen in Fig.1, node $v_{j}$ can be one-hop communicated to node $v_{b}$, but it two-hops communicates. Although energy consumption of "the neck of a bottle" node $v_{b}$ is reduced through tree change, but energy consumption in entire network is increased.

Although the lifetime of node being died is a lifetime of network in previous algorithms, we are going to adapt tree later keeping energy of entire network. So although some nodes consume energy extremely at the present time, since setting such nodes as leave nodes after adaptation, this operation is done in order to keep energy of network.

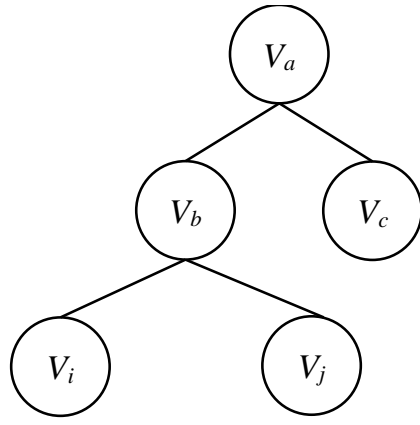

(a) Current state

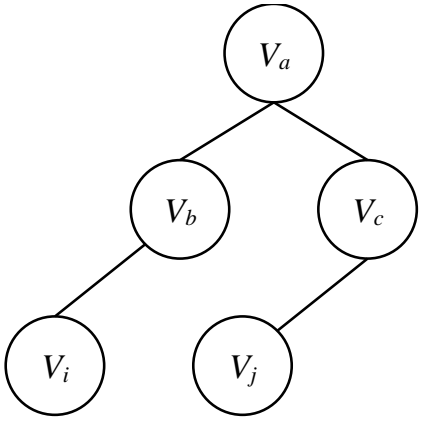

(b) Changed state
Fig.1. Energy consumption of "the neck of a bottle" node $v_{b}$ is reduced through tree change, but energy consumption in entire network is increased

As a result, the tree with at most 3 degree of nodes is optimal [2], the tree with at most 3 degree of nodes is optimal using algorithm proposed in this paper.

Step 2: if it cannot find out $v_{j}$ that is adjacent to all children nodes $v_{i}$ of "the neck of a bottle" node $v_{b}$, but is not children node of $v_{b}$, finish the algorithm.

The proposed algorithm in this paper is simple compared with the previous methods.

Because movement of nodes is short compared with in the previous algorithm, since the tree constructing by the proposed algorithm is a local quasi-optimal tree, not a local optimal tree.

As referred above, adapting a local quasi-optimal tree reaches a tree with maximum lifetime, since this tree is superior to a local optimal tree by the previous algorithm in terms of energy of entire network. As a result, approximation algorithm is as following.

Step 1: calculate weights of all nodes in $T$ creating random tree $T$.

Step 2: classify all nodes into three categories; $V_{1}, V_{2}, V_{3}$ according to weights.

Step 3: reduce the number of children nodes about "the bottle of neck" nodes belong to $V_{1}$. If reducing can be run, calculate weights of all nodes belong to a changed tree $T^{\prime}$ and repeat step 2 and 3 .

Step 4: if reducing weights of "the bottle of neck" nodes cannot be done, output a Tree T.

\subsubsection{Adaptive Change of Tree and Construction Maximum Lifetime Tree:}

The problem of construction maximum lifetime tree can be divided into two problems as following.

First, designing the tree so that the lifetime of node being died first is maximum. This has aimed in previous works. Second, satisfying first and minimizing energy consumption of entire network. This is proposed in this paper. Denoting above two methods mathematically, it is as following.

$$
\begin{gathered}
\arg \max _{T \in T(S)} \min _{i} L(T, i) \\
\arg \max _{T \in T(S)} \min _{i} L(T, i) \text { and } \arg \min _{T \in T(S)} \sum_{i=1}^{N} E_{c o n}^{l o s}(i)
\end{gathered}
$$

It is difficult to optimize two problems in Eq.(24) at the same 
time.

In this paper, algorithm is designed so that quasi-optimal solution, not optimal solution is obtained for the first condition and the second condition is satisfied. Although the tree by this algorithm is optimal in terms of energy of entire network, nodes being died soon are happened due to large number of nodes. Overcoming this, in order to increase more than the lifetime of entire network by step 1, tree is adapted as following.

Step 1: calculate energy consumption of every node per period in a quasi-optimal tree $T$ created first, $E_{c o n}^{\text {los }}(i)$ in the sink. Energy that leave nodes consume is calculated at the same time. After constant $\mathrm{m}$ periods, if following residual energy of "the bottle of neck" nodes reaches constant threshold, a tree is adapted and changed.

$$
\min _{i=1, N} E_{i}^{(m)}=E_{i}^{(0)}-m \cdot \max _{i=1, N} E_{c o n}^{l o s}(i)
$$

Adapting threshold is decided as following.

First, calculate following equation.

$$
M=\frac{\sum_{i=1}^{N} E_{i}^{(0)}}{\sum_{i=1}^{N} E_{c o n}^{l o s}(i)}
$$

$M$ is the number of average rounds that the nodes of network can be alive. After $m$ rotations, if following relative Eq.is satisfied, a tree is adapted and changed.

$$
\begin{aligned}
& \varepsilon+(M-m) \min _{i=1, N} E_{c o n}^{l o s}(i)>\min _{i=1, N} E_{i}^{(m)} \\
& =E_{i}^{(0)}-m \cdot \max _{i=1, N} E_{c o n}^{l o s}(i) \geq(M-m) \min _{i=1, N} E_{c o n}^{l o s}(i)
\end{aligned}
$$

where $\varepsilon>0$ is a parameter deciding whether adapt or not, is below $\min _{i=1, N} E_{c o n}^{l o s}(i)$. In practice, it is convenient to set $\varepsilon=\min _{i=1, N} E_{c o n}^{l o s}(i)$.

Step 2: adapt a tree $T$ as following.

Since the nodes that were "the bottle of neck" nodes in current tree $T$ should be leave nodes, new random tree $T_{\text {rand }}$ is created in entire path as following.

First, choose one-hop neighbor node of the sink $v_{0}$ as the children nodes of $v_{0}$, these are called the first level nodes.

Second, about all neighbor nodes those are not nodes of $V_{0}$, choose all neighbor nodes except for the sink and the first level nodes as its children nodes. Children nodes being shared belong to the node of which residual energy is larger. The children nodes of the first level nodes are called the second level node.

Third, the second level nodes that are not nodes of $V_{0}$ choose all neighbor nodes that are not nodes of one, the second level nodes as their children. Children nodes being shared choose their parent nodes like in above.

Forth, $i^{\text {th }}$ level nodes that are not nodes of $V_{0}$ choose all neighbor nodes that are not nodes of $j^{\text {th }}(\leq i)$ level nodes as their children. Children nodes being shared choose their parent nodes like in above.

Fifth, repeat like in above, output a tree to which all nodes in network belong.

Input a random tree created $T_{\text {rand }}$, quasi-optimal tree creating algorithm. At this time, their parent nodes in $T_{\text {rand }}$, since the nodes of $V_{0}$ cannot be parent nodes.

After finishing algorithm, output a new tree $T^{(1)}$ and operate the network using this tree.

Step 3: calculate energy consumption $E_{c o n}^{\operatorname{los}(j)}(i)$ of all nodes per one period in a new tree $T^{(1)}$ in the sink.

Calculating average number of live rounds as following, after $m^{(1)}$ rounds, if following relative Eq.is satisfied, a tree is adapted and changed.

$$
\begin{gathered}
M^{(1)}=\frac{\sum_{i=1}^{N} E_{i}^{(0)}(1)}{\sum_{i=1}^{N} E_{i}^{(1)}(i)} \\
\left(M^{(1)}-m^{(1)}+1\right) \min _{i=1, N} E_{c o n}^{l o s}(i)>\min _{i=1, N} E_{i}^{(m, 1)} \\
\geq\left(M^{(1)}-m^{(1)}\right) \min _{i=1, N} E_{c o n}^{l o s(1)}(i)
\end{gathered}
$$

where, $E_{i}^{(m, 1)}$ is energy of node $v_{i}$ after m periods after adapting and changing one time.

The Eq.(28) means that "the bottle of neck" nodes in a tree $T^{(1)}$ have residual energy enough to be used as only leave nodes.

If above Eq.is satisfied, "the bottle of neck" nodes in a tree $T^{(1)}$ are added to a set $V_{0}$ and a tree is adapted and changed.

Step 3: A tree $T^{(i)}$ is created running algorithm under limited condition, after creating a random tree $T^{(i)}$ in the same way in step 2.

Step 4: Calculate energy consumption $E_{c o n}^{\operatorname{los}(i)}(j)$ of all nodes per one period in a new tree $T^{(i)}$ in the sink.

Calculating average number of live rounds as following,

$$
M^{(j)}=\frac{\sum_{i=1, N}^{N} E_{i}^{(0)}(j)}{\sum_{i=1}^{N} E_{c o n}^{l o s(j)}(i)}
$$

where, $M^{(j)}$ is average number of live rounds of a tree $T^{(j)}$ created after jth adaptation and change, $E_{i}^{(0)}(j), E_{c o n}^{\operatorname{los}(j)}(i)$ : initial energy and energy consumption during one period of node $v_{i}$ in a tree $T^{(j)}$ created after $\mathrm{jth}$ adaptation and change

After $m^{(j)}$ rounds, if following relative equation is satisfied, a tree is adapted and changed.

$$
\begin{gathered}
\left(M^{(j)}-m^{(j)}+1\right) \min _{i=1, \bar{N}} E_{c o n}^{\operatorname{los}(j)}(i)>\min _{i=1, \bar{N}} E_{i}^{(m, j)} \\
\geq\left(M^{(j)}-m^{(j)}\right) \min _{i=1, \bar{N}} E_{c o n}^{\operatorname{los}(j)}(i)
\end{gathered}
$$

where $E_{i}^{(m, j)}$ is the residual energy of node $v_{i}, m^{(j)}$ periods later, after adaptation and change $j$ times. The Eq.(30) is identical to Eq.(31) in the sense.

Step 5: If Eq.(31) is satisfied, "the bottle of neck" nodes in a tree $T^{(j)}$ are added to $V_{0}$ and a tree is adapted and changed.

Step 6: repeating step 5, if one of following conditions is satisfied, it is considered that the network is died.

- in case of $1 \geq M^{(j)}>0$

- in case that there are all nodes of the network in $V_{0}$ 
- in case that the tree cannot be constructed no more with limited transmission range of nodes.

In most cases, the network is died according to the third condition. That is, if nodes in $V_{0}$ are concentrated on certain area, some of network is died, since it is difficult to construct a tree there.

Compared with this, the cases that the network is died according to the first and second condition can be ideal. So, a tree is created so that the positions of nodes adding to $V_{0}$ is distributed evenly entire network.

This problem causes necessity that should combine topology information of the network in construction maximum lifetime tree. There is no need to consider energy consumption and computation amount in the proposed paper. Because a tree is adapted in the sink. Also, it is almost similar to existing algorithm and computation complexity on running once is simpler than the previous methods, although, it is run several times for adaptation.

\section{RESULTS AND DISCUSSION}

As seen in Fig.2 and Fig.3, latency and energy performance of the proposed tree is much better than MST and SPT. MST is a tree with at most 3 degree of nodes and active time in children nodes belong to one parent is continuous so that the parent nodes are not in idle listening to receive data from children nodes. This tree is constructed in case with aggregation. On the other hand SPT is a tree with the largest depth constructing along the minimum path through avaricious method without considering aggregation. So, latency of MST is shorter than one of SPT with aggregation. The proposed tree is a tree with at most 4 degree of node and the depth of tree is the smallest compared with MST and SPT in same size of WSN. Thus, latency is small relatively.

Also, it can be seen in Table. 1 that the lifetime of network can be increased by almost two times through adaptation. Because the time for the first node of tree to be died is only half of the time for half of nodes in network to be died. In ideal case, the nodes in network almost are died at the same time through the proposed method. As a result, the lifetime of tree is increased by about two times.

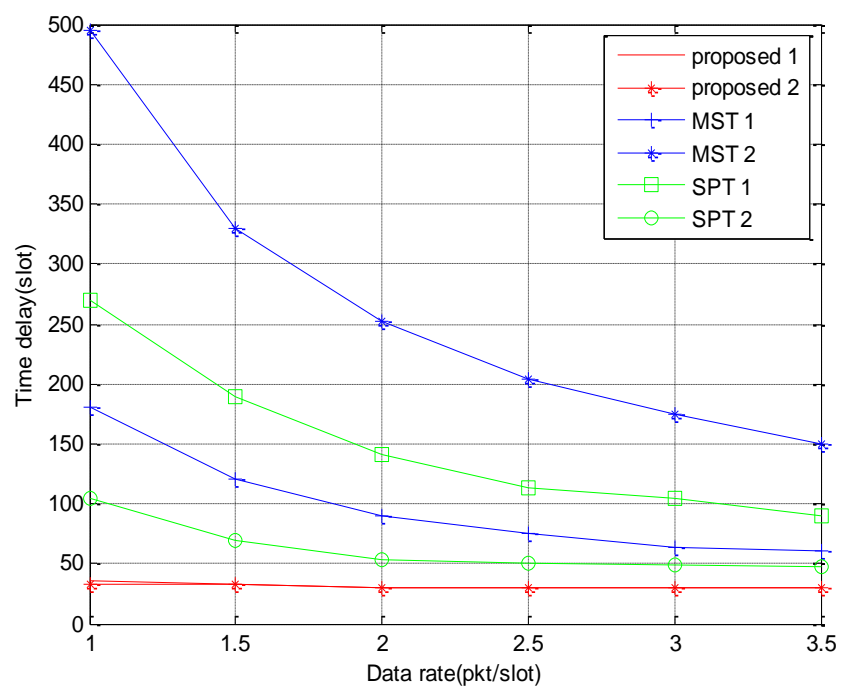

Fig.2. Time delay versus data rate (32 nodes in $100 \mathrm{~m} \times 100 \mathrm{~m}$ area, transmission range $20 \mathrm{~m}$, interference radius $40 \mathrm{~m}$ )
The performance of MST, SPT and the proposed tree all depends on initial tree establishing. Here random same directional tree has been selected as initial tree. Because the performance of the proposed tree depends on initial tree establishing, i.e. the proposed method is of superior performance, only if routing nodes of initial tree are distributed evenly to entire network. Although a random same directional tree is a random tree, here routing nodes of initial tree are distributed evenly to entire network.

Table.1. The lifetime of node according to the protocol. (The number of nodes 100 , sensing area $100 \times 100$ )

\begin{tabular}{|c|c|c|c|c|}
\hline $\begin{array}{c}\text { Energy } \\
\text { (J/node) }\end{array}$ & Protocol & $\begin{array}{c}\text { FND } \\
\text { (First } \\
\text { Node } \\
\text { Dies) }\end{array}$ & $\begin{array}{c}\text { HNA } \\
\text { (Half of } \\
\text { the Nodes } \\
\text { Alive) }\end{array}$ & $\begin{array}{c}\text { LND } \\
\text { (Last } \\
\text { Node } \\
\text { Dies) }\end{array}$ \\
\hline 0.5 & LEACH & 198 & 402 & 519 \\
\hline 0.5 & PEGASIS & 463 & 875 & 1052 \\
\hline 0.5 & ZigBee & 526 & 960 & 1154 \\
\hline
\end{tabular}

As seen in Fig.3, energy consumption per one bit versus data rate is reduced.

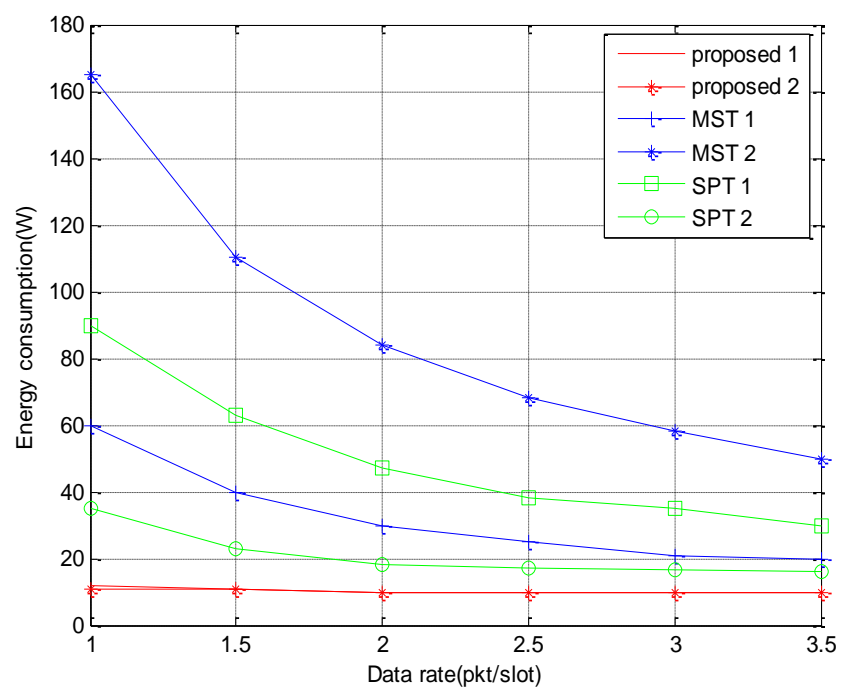

Fig.3. Energy consumption versus data rate

Because the larger data rate, the more additional energy consumption on control messages necessary to transmit data and latency is reduced. The Fig. 4 and Fig.5 shows time delay and energy consumption versus the number of nodes. As seen in figures, the performance of the proposed method is better compared with MST and SPT. Although transmission delay itself is small, latency in nodes is large, since MST and SPT are trees designed for minimum path basis.

Since MST and SPT become trees passing through more nodes in order to reach minimum path basis, total latency is increased due to exchange of control messages and latency in nodes. However, latency is almost constant according to the number of nodes in the proposed

Energy consumption in SPT is increased according to the number of nodes suddenly. Because the larger the number of nodes, the larger the number of hops suddenly, since one of nodes transmit to the nearest node from it. Since the largest degree of tree is limited with 3 in MST, this problem does not happen. In 
terms of energy consumption according to the number of nodes, the proposed method is more robust than MST, since the largest degree of tree is limited with 4 in the proposed method.

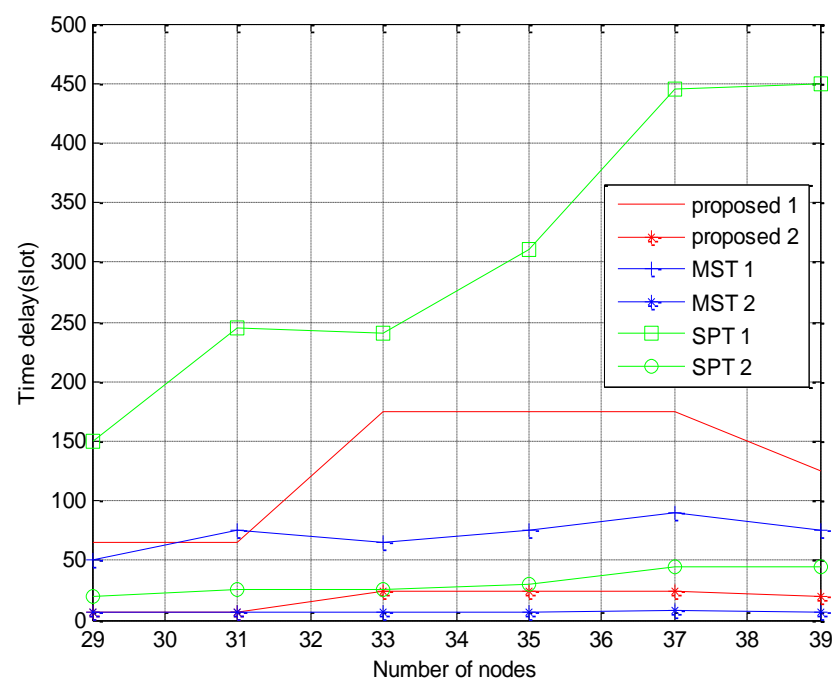

Fig.4. Time delay versus the number of nodes

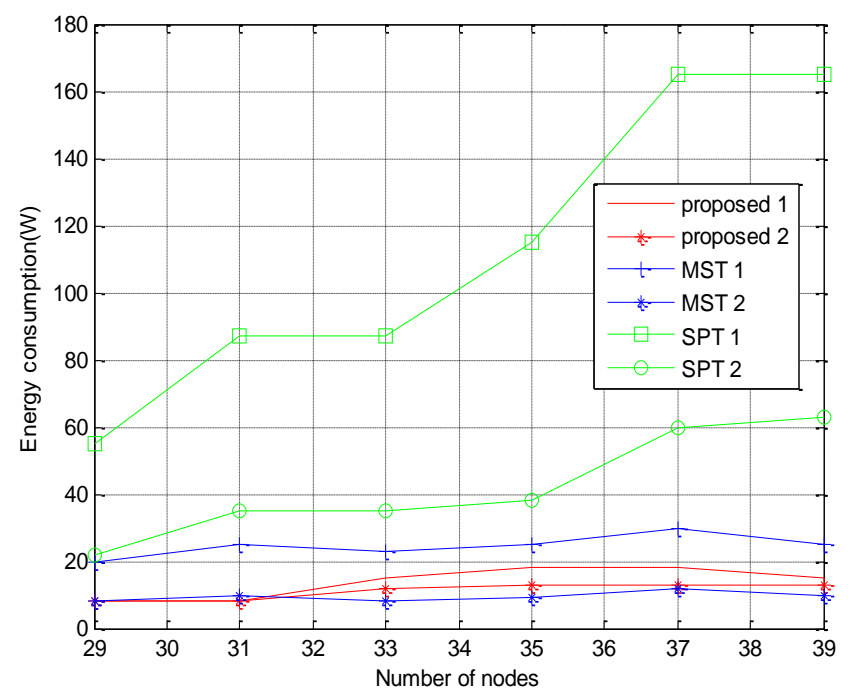

Fig.5. Energy consumption versus the number of nodes

\section{CONCLUSION}

In this paper, we propose energy efficient construction tree in
WSN. Construction maximum lifetime tree can be divided into two categories: with aggregation and without aggregation. But in practice, there are data messages with data aggregation and control messages without data aggregation. Packet loss is not considered in construction maximum lifetime tree in most of previous works, but in practice it reaches average $30 \%$. In this paper, first, we propose the method of construction maximum lifetime tree considering general type of data aggregation, exchange of control messages and packet transmission loss. Second, we propose the method increasing lifetime of tree and reducing complexity and latency combining optimization of energy consumption in entire network through quasi-optimization of local nodes and adapting. Third, we analyze and evaluate the validity of the proposed methods.

\section{REFERENCES}

[1] Kazem Sohraby, Daniel Minoli and Taieb Znati, "Wireless Sensor Networks: Technology, Protocols, and Applications", John Wiley and Sons, 2007.

[2] Junbin Liang, Jianxin Wang, Jiannong Cao, Jianer Chen and Mingming Lu , “An Efficient Algorithm for Constructing Maximum Lifetime Tree for Data Gathering without Aggregation in Wireless Sensor Networks", Proceedings of IEEE INFOCOM, pp. 1-5, 2010.

[3] B.A. Alyoubi and I.M. El Emary, "The Zigbee Wireless Sensor Network in Medical Applications: A Critical Analysis Study", Journal of Current Research in Science, Vol. 4, No. 1, pp. 1-7, 2016.

[4] Y.F. Wen and F.Y.S. Lin, "Energy-Efficient Data Aggregation Routing and Duty-Cycle Scheduling in Cluster-based Sensor Networks", Proceedings of $4^{\text {th }}$ IEEE Consumer Communications and Networking Conference, pp. 95-99, 2007.

[5] L.A. Villas, A. Boukerche, H.S. Ramos, H.A.F. De Oliveira, R.B. De Araujo and A.A.F. Loureiro, "DRINA: A Lightweight and Reliable Routing Approach for in-Network Aggregation in Wireless Sensor Networks", IEEE Transactions on Computers, Vol. 62, No. 4, pp. 676-689, 2013.

[6] H. Yetgin, K.T.K. Cheung, M. El-Hajjar and L.H. Hanzo, "A Survey of Network Lifetime Maximization Techniques in Wireless Sensor Networks", IEEE Communications Surveys and Tutorials, Vol. 19, No. 2, pp. 828-854, 2017. 